\title{
Recombinant B. pertussis Adenylate Cyclase Toxin-Tyrosinase A2 Epitope Vaccine
}

National Cancer Institute

\section{Source}

National Cancer Institute. Recombinant B. pertussis Adenylate Cyclase Toxin-Tyrosinase

A2 Epitope Vaccine. NCI Thesaurus. Code C96735.

A recombinant vaccine containing a genetically detoxified adenylate cyclase toxin (CyaA)

of Bordetella pertussis coupled, through its catalytic site, to the melanoma tyrosinase A2 epitope YMDGT MSQV, with potential antineoplastic activity. Via the toxin moiety, the recombinant $B$. pertussis adenylate cyclase toxin-tyrosinase A2 epitope specifically binds to the alphaMbeta2 integrin (CD11b/CD18) located on CD11b-positive antigenpresenting cells (APC). Upon processing and presentation of the melanoma-specific epitope by MHC class I molecules to the surface of these APCs, a specific cytotoxic T-cell $(\mathrm{CTL})$ response against tumor cells expressing tyrosinase may be initiated, resulting in decreased tumor growth and cell lysis. 